\section{Telomere stutters}

\section{superfluous}

Genes Dev. 17, 2271-2282 (2003)

Telomeric repeats - stretches of recurring DNA sequences - are an integral part of the telomeres that cap the ends of chromosomes. But Mahito Sadaie et al. have shown that chromosomes can work pretty well without them.

Researchers have found before that fission yeast survive when their telomeric repeats are deleted by fusing the yeast's linear chromosomes into circles. Now Sadaie et al. show that one key function of telomeres is also maintained. Telomeres are important during meiosis - the type of cell division that generates reproductive cells - in that they cluster together, thereby allowing chromosome pairing. But Sadaie et al. find that the repeat-less circular chromosomes can still pair up. Moreover, Taz1, a protein that binds telomeric repeats, remains latched onto the repeat-less chromosomes. The group suggests that a genetic sequence in a region called the subtelomere serves as a back-up mechanism to recruit such telomeric proteins.

But telomeric repeats are needed for something. Cells lacking repeats cannot rebuild fully working telomeres after they are dismantled, as the authors showed by creating mutants lacking Tazl and then resupplying the protein. This shows that the three-dimensional structure of the repeats may provide a scaffold for Tazl and other proteins.

Helen Pearson

\section{Atomic physics}

\section{Squishy ball lightning \\ Appl. Phys. Lett. 83, 2283-2284 (2003)}

Ball lightning poses an irresistible puzzle. Nikola Tesla and Peter Kapitsa are among those who have previously sought to find explanations for these glowing balls, typically about the size of a child's head, that float through the air during thunderstorms and sometimes pass through closed doors and windows. They are widely thought to be balls of plasma, and might be akin to the luminous plasmas occasionally formed in the detonation of explosives.

One of the most striking and unexplained properties of these ghostly apparitions is their cohesion. If confined in glass tubing, explosion plasmas can be guided around sharp corners, whereas ball lightning can squeeze through narrow openings. J. J. Gilman suggests that this cohesion might arise in a plasma of Rydberg atoms, which are atoms with an outer (valence) electron that has been excited into an orbital with an extremely high quantum number $\left(10^{3}-10^{4}\right)$. The radius of such an orbital may be macroscopic: up to several centimetres. This makes the electron cloud very 'floppy', so that the atom is highly polarizable and experiences strong van der Waals attractive interactions. Gilman estimates that a plasma containing such Rydberg atoms would be a squishy ball with about one-hundredth the cohesive energy per atom of a metal. Philip Ball

\section{Molecular biology Spreading like wildfire}

Science 301, 1545-1547 (2003)

Gene expression in nematode worms can be shut down by a process called RNA interference (RNAi). An injection of double-stranded RNA (dsRNA) that matches a particular gene suffices to silence that gene throughout the worm and in its progeny. Evan H. Feinberg and Craig P. Hunter now suggest how a protein called SID-1 may mediate this ripple effect.

The natural function of RNAi in worms is not certain, but in some other organisms it protects against viral diseases. Viruses often carry their genetic information on a single strand of RNA, but make doublestranded copies when infecting their host. This triggers the host's defences, which somehow stop the RNA from being translated into functional proteins. In worms, such viruses have not yet been identified; nevertheless, researchers exploit RNAi to study gene function. By introducing specific dsRNA sequences, a worm can be seduced to slice up its own RNA and thus silence gene expression.

Previously, Hunter and colleagues showed that the gene sid-1 is essential for spreading the gene-silencing effects of RNAi throughout a worm. Now they find that the SID-1 protein allows cells to soak up dsRNA. Although it is possible that SID-1 facilitates dsRNA uptake indirectly, the authors propose that it forms a channel that allows the intercellular transport of dsRNA by diffusion. Marie-Thérèse Heemels

\section{Behavioural ecology \\ Horns and lifestyle}

Behav. Ecol. Sociobiol. doi:10.1007/s00265-0030672-6 (2003)

Animals display an impressive array of horns and antlers, from the smooth twisted horns of the kudu, to the curved batteringrams of bighorn sheep, to the many-pointed 'racks' of moose (pictured). But behind this diversity lie some common ecological patterns, T. M. Caro and colleagues have discovered.

The researchers carried out a comparative phylogenetic survey of the headgear and habits of 39 species of deer and 124 species of bovid (which include cattle, antelope, sheep and goats). They find that an animal's

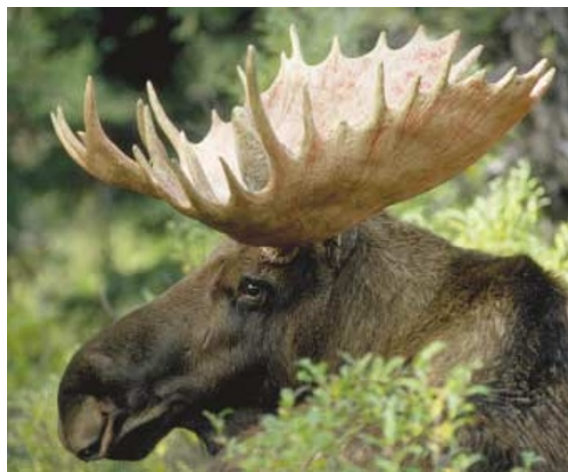

weapons tend to match its social life. Monogamous and solitary species tend to have straight horns whose tips face in, whereas monogamous deer have simple antlers bearing two to five points.

Polygamous, group-living species have a wider range of armaments, which are more likely to be twisted and outward facing. Caro et al. suggest that this reflects the greater competition in social species - between males for mates and females for food - and so the style and intensity of fighting. In contrast, it seems that environment has little influence on horn shape. For example, forest dwellers can apparently possess complex weapons even though they have to dodge through dense vegetation.

John Whitfield

\section{Meteorology}

\section{Oscillating eyes}

Geophys. Res. Lett. doi:10.1029/2003G1017653 (2003)

Chun-Chieh $\mathrm{Wu}$ and colleagues have peered inside Typhoon Zeb, which hit Luzon in the Philippines in 1998, tracking the evolution of its eye. The eye squinted before the storm made landfall, then closed altogether before reappearing wider than ever as the cyclone whirled out to sea - a course of events that the authors have been able to model by computer.

The eye of a tropical cyclone, the calm, windless centre, is surrounded by the eyewall. This is the most intense region, with maximum wind speeds and rainfall, and predicting a storm's course and intensity depends greatly on understanding the eyewall.

Wu et al. studied satellite data of Zeb's behaviour, then modelled it by numerical simulation. As well as simulating the eye shrinking to $50 \mathrm{~km}$ in diameter over the coast of Luzon, they were able to chart its growth to $200 \mathrm{~km}$ with an incomplete eyewall as it headed out to sea, as well as its subsequent narrowing again as the typhoon approached Taiwan.

The aim of this line of work is to improve knowledge of how factors such as ocean temperature and surface terrain influence the development of tropical cyclones, and so help to create better forecasting models.

Tom Clarke 